Revista Herencia Vol. 28 (2), 15-24, 2015

Recibido 31-08-2015 Aprobado 20-09-2015

\title{
BARVA: HISTORIA, URBE Y SOCIEDAD
}

\begin{abstract}
Resumen
En este artículo se hace un abordaje historiográfico, desde la colonia hasta el siglo XX, de acontecimientos que han incidido en la configuración de la morfología social y urbana del Distrito Central de la ciudad de Barva, en Heredia, Costa Rica. Se muestra este espacio como el resultado de la articulación de procesos culturales y socioeconómicos que se plasman en edificios y en el ordenamiento territorial; por esta razón, se recurre al análisis de distintas fuentes documentales para complementar hallazgos y construir una interpretación de la ciudad que trascienda su dimensión material.
\end{abstract}

Palabras claves: historia aplicada, alteridad indígena, arquitectura urbana, historia urbana, control del territorio.

Marcela Otárola

Guevara. Educadora. Profesional independiente. Licenciada en Arquitectura de la Universidad de Costa Rica (1997), Máster en Administración de Empresas con énfasis en Mercadeo Ejecutivo de la Universidad Estatal a Distancia, Costa Rica (2002). Tesis presentada para optar al grado de Magíster Scientiae en Historia Aplicada, Universidad Nacional, Agosto 2015 (Cum laude). La autora agradece a la Dra. María del Carmen Araya Jiménez, a la M.Sc. Lidia Orias Arguedas y al Dr. Carlos Hernández Rodríguez las observaciones hechas a este trabajo, así como su acompañamiento y orientación a lo largo del desarrollo de la investigación. motarolag@gmail.com

\begin{abstract}
In this article a historiographical approach is made, from colonial times to the twentieth century, of events that have influenced the configuration of the social and urban morphology of the central district of the Barva City, Heredia, Costa Rica. This space is displayed as result of joint cultural and socioeconomic processes that are expressed in its buildings and land management; for this reason, it is resorted to analyze different documentary sources to complement findings and build an interpretation of the city that transcends its material dimension.
\end{abstract}

Keywords: applied history, indigenous otherness, urban architecture, urban history, control of territory.

Las ciudades son entes vivos, territorializan dinámicas sociales $\mathrm{y}$, por ende, su morfología cambia. Ellas están sujetas a las contingencias de la época y son el resultado de una cosmovisión que se forja en su devenir y permea el accionar de sus habitantes. Por este motivo, la búsqueda para una comprensión de la fisonomía social y espacial de las urbes conduce a una revisión historiográfica de su fundación como asentamiento y, de esta manera, visibilizar las interacciones que la fraguaron como población.

Con esta premisa, se desarrolló una tesis de investigación en el programa de Maestría en Historia Aplicada de la Universidad Nacional sobre el Distrito Central de Barva, Heredia, Costa Rica. Seguidamente, se presentan hallazgos referentes a procesos históricos obtenidos de crónicas españolas coloniales, registros censales y recopilaciones de estudiosos sobre la historia de Barva y Heredia, que reseñan su institución como poblado.

Descubrimientos como el origen de la designación (y auto designación) de indios que se reproduce oral y textualmente en la localidad, el rol de la Iglesia 
Católica en la construcción física y social del pueblo, el control del uso de la tierra por parte de autoridades políticas en el embrionario emplazamiento y su posterior constitución como una zona agropecuaria; son acontecimientos que edificaron la ciudad y conformaron simultáneamente una identidad en la mentalidad de sus moradores.

De los datos examinados, destacan tres aspectos centrales que contienen ideas arraigadas en valoraciones y rutinas aún conservadas por los ciudadanos barveños. Dichas cuestiones son:

- Fundación de Barva como pueblo de doctrina (S.XVII - S.XIX)

- Separación de Cubujuquí del Valle de Barva (S.XVII - S.XIX)

- Conformación de Barva como zona agropecuaria (S.XIX - S.XX)

\section{Fundación de Barva como pueblo de doctrina (S.XVII - S.XIX)}

Para comprender la pertinencia de este hecho, se debe recurrir a la lectura de textos sobre la colonización de Barva y vislumbrar el pensamiento que prevalecióo. "La visión del conquistador" (Romero, 2011: p. 12) fue el afán que justifico la apropiación española de la tierra y todo lo en ella hubiese $y$, para concretar usufructuar las nuevas posesiones tras la conquista, dieron una identidad socia usufructuar las nuevas posesiones tras la conquista, dieron una identidad soci

Con una meta y una estrategia de dominio definidas, los españoles se instalatégica porque de Barva, asentamiento indigena que ofrecia una posición estrahacia el Mar del Norte (hoy Mar Caribe) a través de una red hidrográfica compuesta por los ríos Joris (Río Sarapiquí), Cutris (Río San Carlos) y el Desaguadero de la Laguna de Nicaragua (Río San Juan), cauces que serían parte de una ruta

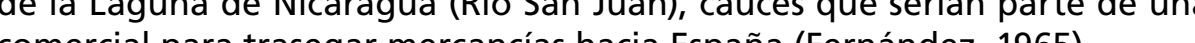

En escritos compilados del siglo XVII se encontró una narración donde se encomienda al alguacil mayor de la ciudad de Cartago y de la gobernación, Jerónimo de Retes, descubrir esa vía y sojuzgar a las poblaciones originarias de la zona:

...de que el capitán Jerónimo de Retes [...] vaya al descubrimiento del dicho puerto y camino, para que se pueda trajinar con requas; y reduzga á los indios sus antepasados y ellos lo habían dado, como son Votos, Güetares y otras naciones, y los haga poblar y pueble en aquella parte y en la que de ellas más cómoda le parezca, para que allí sean industriados y enseñados en las cosas tocantes a nuestra santa fe católica y en toda pulića cristiana... (Fernández, 1965: p. 248) Los españoles se establecieron y legitimaron su permanencia con la fundación
de Barva como un poblado de doctrina y administrado por una orden franciscana que, en 1613, lo llamó San Bartolomé y erigió un convento en él. Luego, en censos tributarios de los siglos XVI y XVII aparece bajo la calificación de pueb/o de indios, es decir como un grupo de indígenas encomendados para tributar a la Corona Española (Fernández, 1975).

Barva figura, entonces, como un conglomerado social productivo, fundado según las ordenanzas españolas y con una localización geográfica ventajosa.
Empero, la iniciativa comercial prevista no se concretó y las condiciones geofísicas, antes favorables, se tornaron en una debilidad. Barreras naturales, largas distancias, además de la ausencia de puentes y caminos, dificultaron la movilización de las personas acentuanto su condición periferica.

Esta situación contrarió a la Iglesia quien vio reducida la participación de feligreses en los actos liturgicos. Ello produjo la instalación en el Barreal mita en calidad de ayuda de una ermita en calidad de ayuda de parroquia fortaleció la interacción de los pueblos focales y redefinió su tejido social (Solecales 1964 ).
ledeinió su tejido social (So-

De lo anterior se colige que la precencia eclesial en la génesis de las constituyó el sitio de concentración constituyó el sitio de concentración el dominio sobre los lugareños.

Esta injerencia fue clara en San Bar-

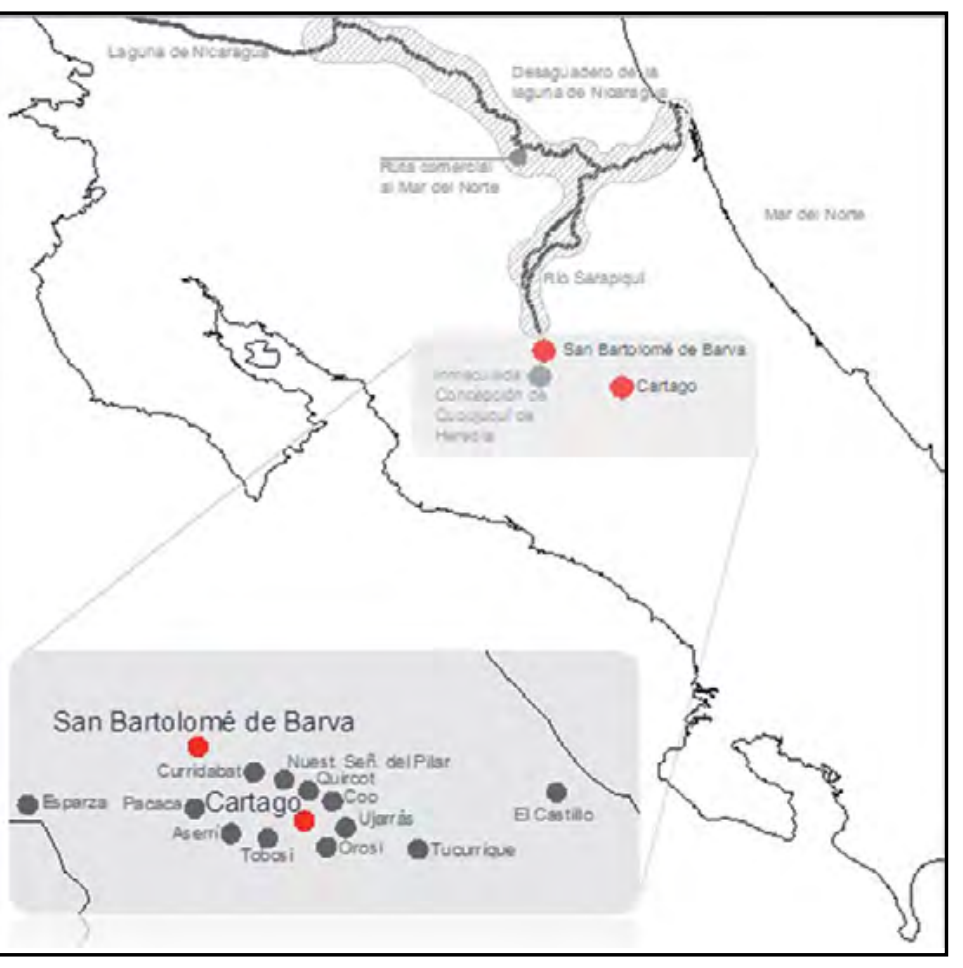

Barva, y documentos analizados así lo manifiestan. Un extracto de un informe al rey de España Fernando IV, redactado por el Obispo Agustín Morel de Santa Cruz, es ejemplo de ello:

El terreno en que se halla es montuoso, tiene iglesia muy capaz, con su coro alto, sacristía, distintas piezas, oficina y claustro cercado de tapias a modo de convento y todas las fábricas son de adobes, y teja. El doctrinero es un franciscano, hay 43 casas de naturales y 10 de ladinos, unas y otras de paja, cuatro calles mal formadas de oriente a poniente y las mismas de norte a sur. Hay un Alcalde, Alguacil Mayor, dos Regidores y Fiscal, Sargento Mayor y una compañía de a pie

con 19 soldados y sus oficiales, 55 familias y 186 personas. Pagan tributo anua

173 pesos, cuatro reales y medio. (Solera, 1964: p. 15).

En el documento se aprecia la descripción de una incipiente configuración urbana en un área montañosa, diseñada en cuadrantes delimitados por calles y ocupada con casas. Además, se constata la condición de San Bartolomé calles pueblo de doctrina, administrado por un clérigo que llevaba el registro de la población y sus tributos.

Como resultado de esta coyuntura, la comunidad construyó parte sustantiva de su identidad sobre el adoctrinamiento religioso, derivando en una organización comunal caracterizada por la obediencia a la autoridad por una homogenización que remarcaba su ascendencia indígena, y por el ejercicio de oficios (la mayoría agrícolas) en calidad de subordinados. Para mantener este dominio, la Iglesia ejecutó prácticas urbanas para la cohesión social. Fue así que promovió la participación de los parroquianos en el ornato y la decoración del templo, organizó donaciones para financiar eventos litúrgicos y coordinó voluntarios en actividades de evangelización; faenas que facilitaron
Figura 1. Ubicación de Costa Rica. siglo XVIII. 
la socialización y se realizaron en espacios públicos: el templo y sus jardines la plaza y las calles aledañas; construyéndose un lugar de gran valor simbólico donde la devoción religiosa primó.

Así, las acciones coloniales realizadas para aprovechar la riqueza de las tierras, depararon también en la formación de un centro urbano primigenio que se mantuvo en siglos subsiguientes.

\section{San Bartolomé y su relación con Cubujuquí}

Tanto San Bartolomé como Cubujuquí se asentaron en el llamado Valle de Barva, "a un cuarto de legua" de distancia entre ellas (Solera, 1964: p. 15) y tuvieron un rol importante en la producción agropecuaria de la provincia. Sin embargo, a pesar de esta cercania, durante el siglo XVII, su desarrollo tomó direcciones distintas y estuvo orientado por la naturaleza de los actores sociales que mediaron ensu definición jurisdiccional. Ellos mantuvieron una lucha entre si por la ración y explotacion de rén sos naturales que decantó en su posterior separación y en una confrontación que impactaria la cor

Los moradores de Cubujuquí fueron colonos españoles y mestizos que llegaron a la zona desde el siglo XVII, para desarrollar actividades agropecuarias productivas como la cría de ganado vacuno y equino. La vocación pecuaria de la zoscansar mulas trá́das desde Nicaragua. Dadas las buenas condiciones para descanar mulas traidas desde Nicaragua. Dadas las buenas condiciones para este uso, se forse rénín por consiguiente fundaron estancias para atonderesta actividad, consolidándola y favoreciendo, a la vez, la economía de la provincia actividad, consol

Las características de estos pobladores, en 1760, las revela el Sargento Mayor don Bentura [sic] Sáenz de Bonilla, Teniente de Gobernador [sic] y Justicia de Cubujuquí y Valle de Barva, en un texto acopiado por el historiador León Fernández (1976, p.195) donde dice:

... cómo al presente está poblada y congregada en esta dha. Población de $\mathrm{Cu}$ bujuquí todas las gentes moradoras del dicho valle de Barba, nobles y plebeyos, quienes con gran celo y eficacia se han esforzado à fabricar sus casas de teja con la Sta. Iglesia todas de teja, y corriendo la casería pajisa de las cuatro cuadras para fuera, siguiendo el mismo orden en las cuadras y calles que las referidas para fuera, siguiendo el mismo orden en las cuadras y calles que las referidas casas de teja y continuandom con todo esmero, que según se promete se puede todos concurren al aumento del culto divino y adelantamiento de esta Sta. Iglesia Parroquial, allende que el referido congreso pasará de más de ochocientas familias, poco más ó menos inclusive en ellas más de ciento cincuenta de gente noble, quienes y cada uno tienen sus fincas de bienes raíces y ganados vacunos y caballares con que mantienen sus familias, ayudados de el comercio que á menudo tienen en la Provincia de Nicaragua con los géneros y espesias que esquilman sus dchas. fincas.

Del relato se deduce que gran parte de los residentes de Cubujuquí eran españoles o sus descendientes, propietarios de la tierra y demás medios de producción. Se infiere también, que en el sitio se formaría un orden basado en estratificación socio racial, expuesto en las viviendas congregadas "debajo de la campana" (Dobles \& González, 1934: p.169), particularmente en su tipificacion, ordenamiento y en el uso de los materiales de construcción, pues las de mayor jerarquía y cubiertas con teja (correspondientes a los poseedores de la riqueza y nexos comerciales) circundaban la Iglesia, en tanto que las de menor rango que casas de jorn pajizo (por lo general casas de jornaleros, artesanos y permás lejos del templo. La jos del templo.

La Iglesia de Cubujuquí se erigió ba 1722 porque su predecesora estaconstruirse una "de median aspecto y decencia" (Fernández, 1976: p. 169). Secificó una de tipo parroquial 169). (7734 fue declaro pa roquialque, dependiente bajo el nombre de Inmendiente bajo elán parroquia in- la quí. En ella se desarrolló el trazo de quí. En ella se desarrolló el trazo de la

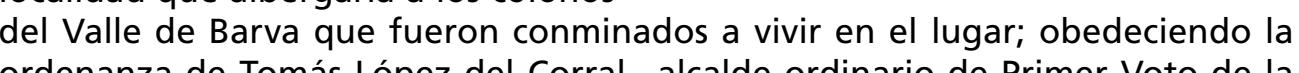
fel Corral -alcalde ordinario de Primer Voto de la Culaderes necesaria dad el título de villa. Este objetivo fue logrado en 1763 cuando don Alonso Fernández de Heredia, Gobernador y Capitán General del reino de Guatemala, otorga la designación bajo el nombre de "Villa de la Ynmaculada [sic] Concepción de Cubujuquí de Heredia" (Fernández, 1976: p. 240).

Durante el proceso de gestión de esta denominación surgen importantes relatos que develan la pugna entre los habitantes de Cubujuquí y de San Bartolomé, porque éstos alegaron que los primeros usurparon sus terrenos, quitándoles espacio para trabajar. En autos de la época se puede encontrar esta denuncia:

Los hijos naturales, principales y común del pueblo de San Bartolomé de Barba, en la Prova de Costa Rica [...] decimos que sin faltar al cumplimiento de ser trivutarios de S.M y uzando de la libertad que nos es concedida de poder apelar al tribunal de V.A, lo hacemos en la forma que sigue: Primeramente ponemos presencia de V.A un punto que tenemos en nuestras ordenanzas, que es la veinte y siete, que ordena de que no pueden vivir los Españoles, mestisos ni mulatos en los pueblos ni en los exidos de ellos por el perjuicio que causan por lo damos cuenta á su Altesa M.P.S. que está fundada la Villa de Cubujuquí dentro de las tierras del dho.ntro.pueblo, que es dentro la legua que nos nombra S.M. (Que Dios guarde), como consta en la ordenanza dha.arriba, y fuera de dicha ViIla otras muchas casas avecindadas del pueblo, todos con sus sercos y cañaverales y platanares, de todos los cuales recibimos muchos perjuicios, como es quitando los montes para hacer sus labores y juntamente cortando las maderas de ellos para hacer otros sercos y dejándonos sin lugar para trabajar nuestras milpas para
Figura 2. Primer esbozo ito central de Barva, siglo XIX. 
poder pagar los Reales Trivutos de S.M., disiendo que no tenemos tierra, que las tierras son dueños de ellas y que se pondrán donde quisieren sin que nadie se los estorve, y de esta suerte se van poblando más y más cada día... (Fernández, 1976: p. 169)

En ese documento, también se encuentra el descargo de la contraparte en la querella. Casimiro Estevan de Arria, procurador del vecindario de la población con el siguiente argumento:

... la población de mis partes no se haya, como los indios suponen, dentro de la legua de resguardo, sino á distancia de tres mil ciento y sesenta varas medidas desde la puerta de la Yglesia hasta la población de mis partes, como lo acredita la certificación dada en quince del pasado Diciembre por el Capn. Dn.Jose Ant. de Oriamuno, Tente. de Governr. y subdelegado de tierras en aquel distrito[.... y componiendo solas dos mill y quinientas varas las cincuenta cuerdas o media legua que corresponde por exido á los pueblos por cada rumbo, resulta que la población de mis partes se hay cituada a distancia de por consiguiente falcificado el informe de sus naturales en esta pay queda poriuicios representados por los naturales de Barba en el cota parte [.... los y sus maderas, con que supos impedirseles las labores de sus milpas, son y sus maderas, con que suponen impediseles las labores de sus milpas, son del Norte de su pueblo se hayan los vestigios de primitiva fundación parté e Norte de su pueblo se hayan los vestiglos de primitiva fundación, es a saber, los cimin tes de mana que no tienen otro límite que la ribera del mar... (Fernán cor1976: p. 235) Los alegatos de los indígenas se consideraron insultos por los habitantes de cubujuqui, quienes, conscientes de su ascencion por dejar clara su condicion comtemente su trajajo: características opuestas a ellos: ciudada de ejercer eficostumbres y con proba capacidad para una exitosa repoblación (Fernández 1976) Consecuentemente con esta perspectiva se continuó la expropiación de campos indígenas y se robusteció una relación de poder sustentada de campos indigenas y se robustecio una relación de poder sustentada en la desiguales, una vez refrendados por diversos reglamentos.

Con el otorgamiento del título de villa a Cubujuquí, ésta se separa del Valle de Barva y, con ello, la situación de alteridad se haría más evidente pues quedarían determinados los perfiles de las dos localidades: Cubujuquí se concebirá como el afincamiento de una élite económica y social superior; mientras que San como el afincamiento de una élite economica y social superior; mientras que San civilizados, dentro de una percepción en la que el segundo poblado figurarí como un paraje perimetral del primero.

Al dictarse la división territorial en el siglo XIX, esta situación quedaría ratificada, pues se estableció que el territorio del Valle de Barva pertenecería a la provincia llamada ahora Heredia, la cual tendría dos cantones: Barba y Heredia siendo esta última su cabecera (Dobles \& González, 1934).

\section{Conformación de Barva como un territorio agropecuario (S.XIX-S.XX)}

El advenimiento de la independencia en el siglo XIX ocasionó la organización del Estado costarricense e impulsó la ejecución de políticas de desarrollo económico, siendo el cultivo del cafe de exportación una de ellas. Esta orientación facilito la expansion del grano en varias partes del pais incluyendo a Barva y transformo el uso forestal de su suelo pues, al tornarse primordial la actividad cafetalera en la zona a finales del siglo $X I X$ e inicios del $X X$, disminuyó la pecuaria, la agricola de pequeña escala (maiz y el frijol) y empezó a decrecer la cobertura boscosa.

Este producto requirió, en primera instancia, de terrenos para la siembra y de caminos para su trasiego. Las tierras fueron adquiridas por un grupo dominante, descendiente de antiguos colonos que se beneficiaron con el cambio en la cofradias fueron asumidos por los ayuntamientos (nuevas formas de gobirn colad y se llam asu Fondos de los Pueblos o Fondos Píos que, a igual que los local) y se llamer indígenas), fueron dados en a quiler a aparceros que se convirtieron en sus le gítimos dueños cuando se habilitó su adquisición por particulares mediante la gitimos dún cos cuna ley en 1835 (Bolaños \& Valverde, 1978).

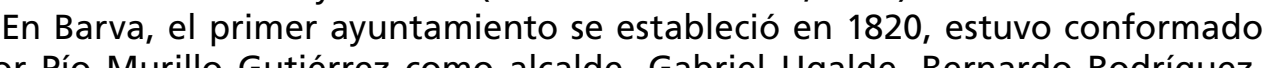
por Pio Muillo Gutierrez coń alcalde, Gabriel Ugalde, Bernardo Rodríguez, te investidos como alcaldes o parientes de otros que detentarín posteriormente investidos como alcaldes o parientes de otros que detantarian esta posición, cioun consta de naturas hipotecaria sobre los Fondos Pís, se detalla los tratantes en estas cestas gestiones (algunos fueran sobre los Fon otros sus (a gunilias y y serevela la que serí el núcleo del poblado. La disposición de estos inmuebles conformaría un anillo perimetra con torno a los espacios públicos de mayor significado para públicos de (la Iglesia y la plaza). En él se establecerían los habitantes con mayor 年 rango y reconocimiento, pues la centro hegemónico de la ciudad era directamente proporcional al escalafón de sus dueños en la esescalafon de sus dueños en la esde una naciente élite política y de una entidad religiosa (arraigada una entidad religiosa (arraigada y en la construcción de proyectos de infraestructura: algunos a través de la acción gubernamental, otros mediante la gestión directa de los

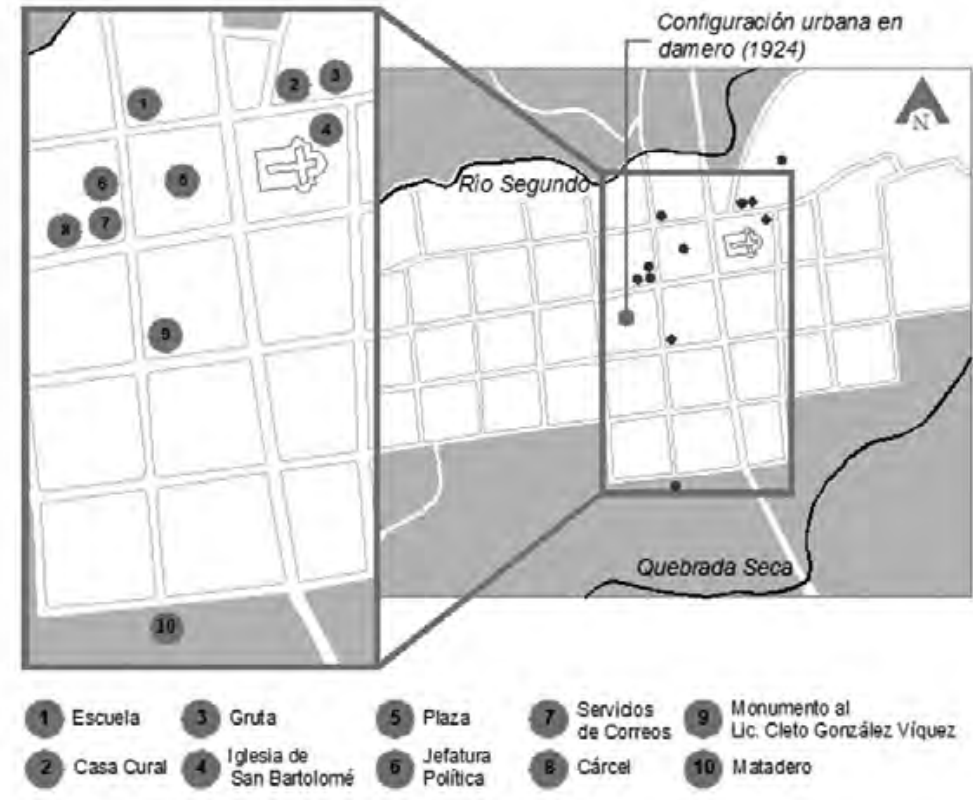

Figura 3. Trazo urbano Berva, siglo XX. . 
vecinos, contabilizando durante este siglo obras edilicias e institucionales que fundarían la base de la actual ciudad (Solera, 1964)

La ordenación de este lugar definió un núcleo de población (no sólo por la construcción de obras sino, también, por la interacción social comportada) que suscitó el surgimiento de pueblos perimetrales, como San Pedro y San Pablo, productores de café y ganadería en gran volumen. La siembra cafetalera fue pródiga y la cosecha del grano se manufacturó en beneficios instalados en esas localidades, donde tambien se cultivó caña de azúcar para el consumo familia y abastecimiento local, requiriendo la construcción de 12 trapiches en el cantó (Archivo Nacional de Costa Rica [ANCR], 1923).

Ante este crecimiento surgio la necesidad de transportar mercancías eficientemente, originando la construcción y mejora de la infraestructura vial, razón por la cual se pavimentaron calles y se reforzaron puentes. De ahi que las vias de comunicación favorecieron la integración entre las poblaciones barveñas, vinculándolas para forjar una zona agropecuaria con un pódulo definido por la concurrencia de rutas y que, anos más tarde, se consolidó por la proliferación de areas residenciales, comerciales, civicas, polticas y religlosas.

La convergencia descrita contribuyó a establecer la cabecera cantonal. En ella a pactir dos, se acrén có la oferta de servicios urbanos, financieros e instituciolocalidades, se acrecento la oferta de senvicios urbanos, finás heros énstitucioefecto la "metropolización" del Valle Central (Carvajal, 2005: p. 53). Se consor e un nodo donde confluyeron los sistemas ideológico, político y ecońs tells, 1974) expuestos en edificios religiosos, municipos y mercontiles, a (Casde expios lúdicos para la interacción comunal como la plaza, el piles, además de la cultura En este ámbito se instauró el locus (Rossi, 1982: p. 185) de Barva: un espacio de enunciación del ser barveño -y por tanto único- que se percibe el paisaje arquitectónico cuando se interpreta como expresión de los modos de vinculación de sus habitantes acaecidos en el tiempo.

\section{Reflexión final}

Los hechos descritos son muestra de que las ciudades recogen dinámicas pasadas y las articulan en un ordenamiento social y espacial. Así, para el caso de de un centro de población y el origen prácticas de cohesión social vigentes. La separación de Cubujuquí del Valle de Barva, presenta una alteridad que destacó el origen indígena de la comunidad que la identifica y perfila mientras que la configuración de una zona agropecuaria expone el proceso de desarrollo y consolidación de su territorio.

En este sentido, el aporte de la historiografía permite abordar la ciudad en dos dimensiones complementarias, una material y otra social, trascendiendo así análisis descriptivo de la urbe y la disertación abstracta de las acciones humanas que ocurren en ella.

\section{Bibliografía}

Archivo Nacional de Costa Rica [ANCR]. (1904). Índice de los Protocolos de Heredia, 1751-1821. San José: Tipografía Nacional.
Archivo Nacional de Costa Rica [ANCR]. (1923). Censo Agrícola e Industrial de 1923.

Blanco, Aragon. (2011). El cambio en las áreas boscosas de San José de la Montaña 1890-2000. Manuscrito no publicado. Escuela de Historia, Universidad Nacional.

Bolaños, M., \& Valverde, N. (1978). Barva y su artesanía tradicional: un legado indígena. San José: Ministerio de Cultura, Juventud y Deportes.

Carvajal, Guillermo. (2005). La organización del espacio urbano de la Ciudad de San José: temas y problemas a inicios del tercer milenio. San José: Editorial Librería Alma Mater.

Castells, Manuel. (1974). La cuestión urbana. México: Siglo veintiuno Editores.

Dobles, L., \& González, C. (1934). La Provincia de Heredia. San José: Imprenta y Librería Lehmann.

Fernández, León. (1965). Colección de documentos para la Historia de Costa Rica. Tomo 2. San José: Imprenta Nacional.

(1975). Historia de Costa Rica durante la dominación española 1502-1821. San José: Editorial Costa Rica.

(1976) Colección de documentos para la Historia de Costa Rica. Tomo 3. San José: Editorial Costa Rica.

Martínez, Héctor. (1977). "Las cofradías en Nueva España". Primer Anuario del Repositorio Institucional de la Universidad Veracruzana. 1, pp. 45-71. Recuperado de http://cdigital.uv.mx/bitstream/123456789/7975/2/anua-Ipag45-71.pdf

Romero, José Luis. (2011). Latinoamérica: las ciudades y las ideas. Buenos Aires: Siglo Veintiuno Editores.

Rossi, Aldo. (1982). La arquitectura de la ciudad. Barcelona: Editorial Gustavo Gili S.A.

Solera, Guillermo (1964). El Cantón de Barba. Apuntes Históricos de su Fundación y Progreso 1562-1964. San José: Imprenta Nacional. 
Revista Herencia Vol. 28 (2), 2015 